\title{
Incidence of Gastrointestinal Perforations in Patients with Rheumatoid Arthritis Treated with Tocilizumab from Clinical Trial, Postmarketing, and Real-World Data Sources
}

Sharareh Monemi · Erhan Berber · Khaled Sarsour · Jianmei Wang •

Kathy Lampl $\cdot$ Kamal Bharucha $\cdot$ Attila Pethoe-Schramm

Received: June 4, 2016/ Published online: July 15, 2016

(C) The Author(s) 2016. This article is published with open access at Springerlink.com

\section{ABSTRACT}

Introduction: The aim of this study was to use multiple data sources to update information on gastrointestinal perforations (GIPs) during tocilizumab (TCZ) treatment in patients with rheumatoid arthritis (RA).

Methods: Reporting rates of GIP events were estimated from three distinct patient data sets: a TCZ-IV RA clinical trial all-exposure population, a global TCZ postmarketing safety database population, and a US healthcare claims database population of patients with RA, including patients who received TCZ, anti-tumor necrosis factor (aTNF) agents, or abatacept.

Enhanced content To view enhanced content for this article go to http://www.medengine.com/Redeem/ B1E4F06017EF20F4.

S. Monemi $(\varangle)$ - E. Berber · K. Sarsour - K. Lampl ·

K. Bharucha

Genentech, Inc., South San Francisco, CA, USA

e-mail: monemis@gene.com

J. Wang

Roche Products Ltd., Welwyn Garden City, Hertfordshire, UK

A. Pethoe-Schramm

F. Hoffmann-La Roche, Basel, Switzerland
Results: The clinical trial, global postmarketing, and healthcare claims populations provided 17,906, 382,621, and 3268 patient-years (PYs) of TCZ exposure, respectively. GIP incidence rates $[95 \%$ confidence interval (CI)] were 1.9 (1.3-2.7), 1.2 (1.1-1.3), and 1.8 (0.7-4.0; specific definition) to 2.8 (1.3-5.2; sensitive definition) per 1000 PYs for the clinical trial, postmarketing, and healthcare claims populations, respectively. The GIP incidence rate $(95 \% \mathrm{CI})$ for the comparator aTNF healthcare claims population ranged from $0.6(0.3-1.2)$ to $0.9(0.5-1.5)$ per 1000 PYs, for an absolute rate difference between TCZ and aTNFs of 1.2 ( -0.3 to 2.5 ) to $1.9(0.0-3.7)$ per 1000 PYs, corresponding to a number needed to harm between 533 and 828 .

Conclusion: The TCZ GIP event rates from multiple data sources were consistent with previously reported rates, did not increase over time, and were significantly associated with the number of prior biologics. Comparison of GIP incidence rates among patients with prior biologic exposure suggests that, for every 1000 patients treated with TCZ per year, an additional 1-2 GIP events might occur compared with patients treated with aTNFs. 
Funding: Roche.

Keywords: Gastrointestinal perforation; Rheumatoid arthritis; Tocilizumab

\section{INTRODUCTION}

Conventional synthetic disease-modifying antirheumatic drugs (csDMARDs) are the first choice of treatment for patients with rheumatoid arthritis (RA); however, for the approximately $50-70 \%$ of patients with RA who do not respond adequately to csDMARDs alone, therapy with a biologic DMARD is recommended [1-3]. Biologic DMARDs currently indicated for RA include five anti-tumor necrosis factor (aTNF) agents, the anti-interleukin 6 receptor therapy tocilizumab (TCZ), the B-cell-targeted therapy rituximab, and the T-cell costimulatory modulator abatacept. The 2013 update of the European League Against Rheumatism (EULAR) recommendations for the management of RA state that in patients with an incomplete response to methotrexate and/or other csDMARDs, biologic DMARDs should be started in combination with methotrexate [4]. The EULAR Task Force regards all currently approved biologic DMARDs as being similarly effective (with the exception of anakinra) and generally safe for use as an initial biological therapy after csDMARD failure. Similarly, no specific biologic DMARD recommendation was made if patients failed their first biologic DMARD; patients should be treated with another biologic DMARD with a similar or different mechanism of action.

TCZ is a humanized monoclonal anti-interleukin 6 receptor antibody; its safety and efficacy have been demonstrated in $>5000$ patients with RA in randomized controlled trials and their long-term extensions, as monotherapy or in combination with csDMARDs [5-13]. Based on these clinical trials, TCZ was approved to treat RA in the European Union in 2009 and in the United States (US) in 2010. It is currently indicated to treat adult RA [intravenously (IV) and subcutaneously (SC)] and systemic- and polyarticular-course juvenile idiopathic arthritis (IV).

A study by Curtis et al. [14] evaluated the overall safety of TCZ in patients with RA using TCZ data from a large postmarketing safety database (cutoff date July 29, 2011) and clinical trials (cutoff date April 1, 2011) and aTNF comparator data from a US external healthcare insurance claims database (January 1, 2010 to June 30, 2014) and published literature. The overall risk of serious adverse events (SAEs) associated with TCZ treatment was not greater than with aTNF agents.

It was also shown in the analyses by Curtis et al. [14] that the rates of certain TCZ-related safety events of special interest-including serious hepatic events, serious gastrointestinal (GI) events, serious myocardial infarctions, serious strokes, and cardiac deaths-were similar to corresponding rates from the aTNF comparator population. Of particular interest, GI perforation (GIP) is a rare but serious adverse drug reaction that has been observed in relation to medications for RA and is also listed under the warnings and precautions of the TCZ prescribing information. Risk factors for GIP in patients with RA include a history of diverticulitis, advanced age, use of glucocorticoids and/or nonsteroidal anti-inflammatory drugs (NSAIDs), and smoking [15-18]. In the TCZ safety analysis, Curtis et al. reported GIP incidence rates [95\% confidence interval (CI)] of 1.5 (1.1-1.8) per 1000 patient-years (PYs) in the TCZ global postmarketing safety database population (up to July 29, 2011) and 2.0 (1.3-2.9) per 1000 PYs in the TCZ clinical trial population (up to April 
1, 2011); the reported GIP incidence rate (95\% CI) in the aTNF US healthcare claims database population was 1.4 (1.1-1.8) per 1000 PYs [14]. However, the variety of sources for event rates limits the ability to compare between the different therapies, and the information regarding GIP associated with TCZ remains limited.

In order to update the currently available information on GIP events in patients with RA treated with TCZ, we examined GIP events from clinical trial and real-world (postmarketing and healthcare claims) populations. In the current study, the frequency of adjudicated GIP events from the TCZ-IV RA clinical trial program population (data cutoff December 2014) and the incidence of GIP events from a postmarketing population in the TCZ global safety database (cutoff date April 10, 2015) were studied. Furthermore, data were obtained from a large administrative healthcare claims database (data collected from January 1, 2010 to June 30, 2014) to assess the rate of GIP events in patients with RA treated with TCZ, aTNF agents (overall and individual agents), and abatacept. The availability of this healthcare claims data, which include the baseline characteristics of the patients, offers the unique opportunity to compare the incidence rates of GIP between patients with RA who received TCZ or other biologics in real-world clinical settings.

The objective of this report was to update the available GIP safety information associated with TCZ administration from multiple data sources (clinical trials database, postmarketing, and real-world administrative claims) and to examine whether the frequency in risk of GIP has changed over time. In addition, the incidence of GIP from a single real-world healthcare claims database was compared between patients with RA who received TCZ, aTNF agents, or abatacept.

\section{METHODS}

Reporting rates of GIP events were estimated in three distinct patient data sets: a TCZ-IV clinical trial all-exposure population database (not including studies performed in Japan), a global company postmarketing safety database population, and a publicly available healthcare insurance claims database population-a retrospective cohort of patients with RA from US healthcare claims databases [Truven Health MarketScan $^{\circledR} \quad$ Commercial Claims and Encounters (commercial) and Medicare Supplemental and Coordination of Benefits (Medicare)].

\section{Clinical Trial Database Population}

The clinical trial database population included patients with RA from the 5 core TCZ-IV Phase III RA clinical trials (OPTION, RADIATE, TOWARD, AMBITION, and LITHE; ClinicalTrials.gov identifiers: NCT00106548, NCT00106522, NCT00106574, NCT00109408, and NCT00106535, respectively) [5-9], the TCZ-IV RA lipid study (MEASURE) [19], the TCZ-IV in early RA study (FUNCTION; ClinicalTrials.gov identifier: NCT01007435) [13], the TCZ-IV monotherapy study (ADACTA; ClinicalTrials.gov identifier: NCT01119859) [20], and the all-exposure data from the TCZ-IV long-term extension RA studies. The data cutoff was December 2014, after the TCZ-IV long-term extension studies were completed.

\section{Global Postmarketing Safety Database Population}

Reporting rates of GIP events in the global postmarketing setting were based on AEs occurring in TCZ-treated patients recorded in the company postmarketing safety database up 
to April 10, 2015. This database records information on all spontaneously reported cases (serious and non-serious) in which TCZ is per default considered "suspect" and all non-interventional program/ non-interventional study cases (serious and non-serious) in which TCZ is considered "suspect"; reports from the literature are also included. All AEs, regardless of whether the reporter assessed it to be causally related to TCZ, were included in this analysis.

\section{Classification of GIPs in the Clinical Trial and Global Postmarketing Safety Database Populations}

Cases were identified by using Standardized Medical Dictionary for Regulatory Activities (MedDRA) Queries (SMQs) ("GIP-narrow") version 18.0 for TCZ cases in the global postmarketing safety and clinical trial databases. Because the "GIP-narrow" events included many non-GIP events, these events were not included in the adjudicated events. All qualifying cases for "GIP-narrow" went through an internal medical review individually in terms of primary reporter type (medically confirmed or consumer cases), concomitant medication use, drug-event latency period, de-challenge and re-challenge information, event outcome, risk factors/contributory factors, and concurrent/ underlying medical conditions known to be associated with GIP. Cases reporting abscess or peritonitis with no evidence of perforation were not considered to be GIP events. Based on the anatomical location of the perforation, all qualifying GIP events were categorized as upper GIPs (esophagus, stomach, duodenum, biliary tract, and gallbladder) or lower GIPs (jejunum, ileum, cecum, colon, rectum, and anal region). Reports citing a small intestinal perforation with no further anatomical details were categorized as "lower" GIP.

\section{Healthcare Insurance Claims Database Population}

GIP incidence rates were calculated using data from an external US healthcare claims database (MarketScan). Adult patients with RA who had evidence of a prescription for or administration of a biologic agent between January 1, 2010, and June 30, 2014, were included: no prior use of the qualifying biologic, continuous enrollment for $\geq 180$ days prior to the index date, both medical and pharmacy benefit plus complete data availability during both baseline and follow-up periods, a minimum follow-up on the qualifying biologic of 30 days, and-for the specified population-evidence of prior use of a biologic other than the qualifying biologic. The index date was the date of fill of the first prescription or administration of the qualifying biologic. Patients with a history of GIP, GI cancer, ulcerative colitis, or Crohn disease during the 12 months prior to the index date were excluded. Patients were followed within each treatment episode until disenrollment from MarketScan, end of the study period, discontinuation of the qualifying biologic, switch to a new biologic agent, or occurrence of hospitalization for GIP.

\section{Safety Analysis in the Healthcare Insurance Claims Database Population}

Two definitions of GIP were evaluated in the healthcare claims databases to generate a best estimate of the range of the incidence rates of true GIP [21]. The first definition (sensitive) included any inpatient admissions with evidence of perforation based on: (1) the presence of the word perforation in the following International 
Classification of Diseases, Ninth Revision, Clinical Modification (ICD-9-CM) diagnosis descriptions: esophageal rupture; gastric, duodenal, peptic, or gastrojejunal ulcer; appendicitis; and GIP of an unspecific location in the large intestine; or (2) an ICD-9-CM diagnosis of diverticulitis, diverticulosis, or ischemic colitis plus a Current Procedural Terminology code for suture or resection of the small or large intestine. The second definition (specific) included only inpatient admissions with evidence of perforation based on the presence of the word perforation in ICD-9-CM diagnosis descriptions for esophageal rupture; gastric, duodenal, peptic, or gastrojejunal ulcers; and unspecified GIP. The specific GIP definition did not include cases of appendicitis, diverticulitis, diverticulosis, or ischemic colitis associated with surgical GI procedures.

Adjusted incidence rate ratios (IRRs) were obtained by using Poisson regression and exponentiating the coefficients. Multivariate adjustment was performed comparing TCZ with aTNF combined. The following baseline covariates were adjusted for in the multivariate models: age, sex, cumulative oral glucocorticoid and NSAID use in the 180 days prior to the index date, history of diverticulitis, number of prior biologics, and observed duration of RA.

This article is based on previously conducted studies, and does not involve any new studies of human or animal subjects performed by any of the authors.

\section{RESULTS}

\section{TCZ-IV Clinical Trial Database Population}

The TCZ-IV all-exposure RA clinical trial population includes data from 5185 patients who received $\geq 1$ dose of TCZ-IV and includes all data from the time of first TCZ dose, representing 17,905.9 PYs of exposure (Table 1). From the SMQ "GIP-narrow" search, 70 events were reported in 53 patients. Of these, 34 events in 31 patients were adjudicated as GIPs, for an incidence rate (95\% CI) of 1.9 (1.3-2.7) events per 1000 PYs. Of these 34 events, 9 occurred in 9 patients (29\%) with a history of diverticular disease, gastritis, or ulcer, and 16 occurred in 16 patients (52\%) with a diagnosis of diverticular disease at the time of surgery or during the course of the clinical trial. There was no increase in number of GIPs with increased TCZ exposure or duration of study (Table 1). The overall incidence rate observed during greatest PY exposure period (>36 months, with 6872.9 PYs of exposure) was comparable with rates observed at earlier time points. The majority of GIPs (85\%) in the TCZ-IV all-exposure population occurred in the lower GI tract.

In the all-exposure TCZ-IV clinical trial population, baseline demographic data were comparable between the overall population and patients who experienced a GIP (Table 2). The mean (standard deviation) age was greater for patients who experienced an adjudicated GIP than for the overall population: 58.5 (10.6) years compared with 51.7 (12.8) years - a trend with age being a risk factor for GIP [17]. The proportion of patients with prior exposure to aTNF agents was $22.6 \%$ for those who experienced an adjudicated GIP compared with $17.0 \%$ for the overall population. Of patients with an adjudicated GIP, 17.9\% had a history of smoking compared with $18.0 \%$ of patients in the overall population.

SC-administered TCZ has been studied in 2 RA clinical trials, with a total of 2039 PYs of exposure $(n=1374)$. The incidence rate of GIP events in the TCZ-SC all-exposure population was slightly lower (1.5 per $1000 \mathrm{PYs}$ ) than in the 


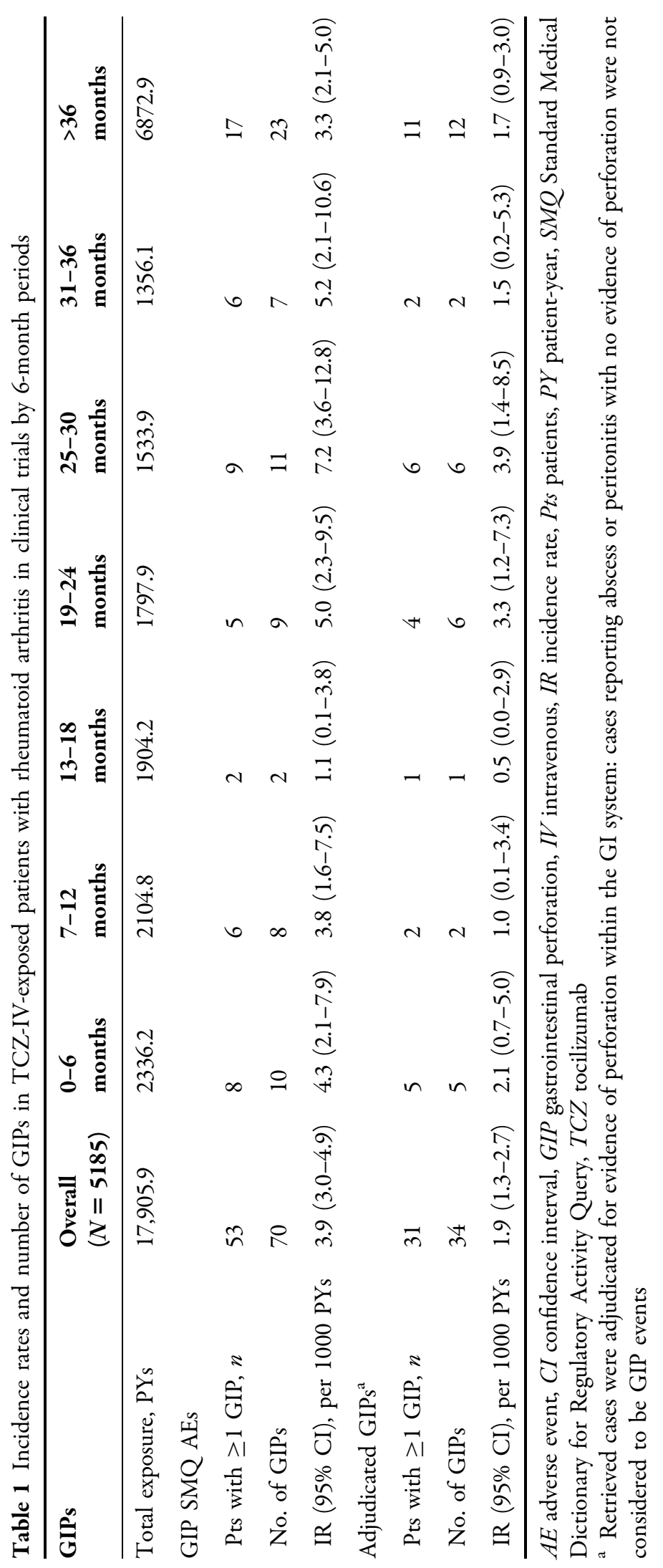


Table 2 Baseline characteristics of TCZ-IV-exposed patients with rheumatoid arthritis in clinical trials

\begin{tabular}{llll}
\hline Characteristics & Overall $(\boldsymbol{N}=\mathbf{5 1 8 5})$ & GIP SMQ AEs $(\boldsymbol{N}=\mathbf{5 3})$ & ${\text { Adjudicated } \mathbf{G I P s}^{\mathbf{a}}(\boldsymbol{N}=\mathbf{3 1})}$ \\
\hline White, \% & 75.0 & 75.5 & 71.0 \\
RF positive, \% & 79.0 & 84.9 & 87.1 \\
Age, mean (SD), years & $51.7(12.8)$ & $57.2(13.4)$ & $58.5(10.6)$ \\
Female, \% & 81.1 & 75.5 & 71.0 \\
Weight, mean (SD), kg & $73.8(18.4)$ & $78.4(16.9)$ & $75.6(15.2)$ \\
Smoker, \% & 18.0 & 26.5 & 17.9 \\
Prior aTNF use, \% yes & 17.0 & 18.9 & 22.6 \\
Baseline DMARD use, \% yes & 66.7 & 64.1 & 83.9 \\
\hline
\end{tabular}

$A E$ adverse event, $a T N F$ anti-tumor necrosis factor, DMARD disease-modifying antirheumatic drug, GIP gastrointestinal perforation, $I V$ intravenous, $R F$ rheumatoid factor, $S M Q$ Standard Medical Dictionary for Regulatory Activity Query, $T C Z$ tocilizumab

${ }^{a}$ Retrieved cases were adjudicated for evidence of perforation within the GI system: cases reporting abscess or peritonitis with no evidence of perforation were not considered to be GIP events

b Percentages were based on patients with nonmissing data. In the overall group, 716 of 3986 patients were smokers; in the adjudicated GIP group, 5 of 28 patients were smokers

TCZ-IV all-exposure population. Given the limited exposure and lack of increased risk, data from the TCZ-SC clinical trials are not included in this analysis.

\section{TCZ Global Postmarketing Safety Database}

The SMQ "GIP—narrow" search retrieved 449 events in 437 patients from the global postmarketing safety database for which a causal or temporal association between GIPs and TCZ could not be excluded, for an overall reporting rate $(95 \% \mathrm{CI})$ of $1.2(1.1-1.3)$ GIP events per 1000 PYs over 382,621 PYs of postmarketing TCZ exposure (Table 3).

Of 295 patients with available information for a comprehensive medical analysis, perforation occurred in the upper GI tract in 36 patients, the lower GI tract in 202 patients, and an unspecified location in 60 patients; 3 patients experienced $>1$ GIP (Table 3 ). Of the 202 patients with a lower GIP, $152(75.2 \%)$ were receiving glucocorticoids, 96 (47.5\%) were receiving NSAIDs, 78 (38.6\%) were receiving both glucocorticoids and NSAIDs, and 17 (8.4\%) were receiving aspirin. Also of the 202 patients with a lower GIP, 135 (66.8\%) had reported diverticular problems, including a medical history in 23 patients $(11.4 \% ; 14$ with diverticulosis, 7 with diverticulitis, and 2 with both diverticulosis and diverticulitis), concurrent diverticular disease in 22 patients (10.9\%; 14 with diverticulosis, 4 with diverticulitis, and 4 with both diverticulosis and diverticulitis; 1 patient had both concurrent and medical history of concurrent diverticular disease), and 91 patients with no medical history of concurrent condition of diverticular disease for whom diverticulosis, diverticulitis and/or diverticular perforation was reported at the time of GIP diagnosis (7 with diverticulosis, 17 with diverticulitis, 24 with diverticular perforation, 5 with diverticular perforation accompanied by diverticulosis, 36 with diverticular perforation accompanied by diverticulitis, and 2 with diverticular 
Table 3 GIPs in patients treated with TCZ reported to the global postmarketing safety database

Patients treated with TCZ

Patients with $\geq 1$ GIP, $n$

Total exposure, PYs

No. of GIP events

No. GIP events per 1000 PYs (95\% CI)

Upper GIP, $n^{\mathrm{b}}$

Concomitant glucocorticoids, $n$ (\%)

Concomitant NSAIDs, $n(\%)$

Both concomitant glucocorticoids and NSAIDs, $n$ (\%)

Concomitant aspirin, $n$ (\%)

Medical history of concurrent peptic ulcer disease, $n$ (\%)

Lower GIP, $n^{\mathrm{b}}$

Concomitant glucocorticoids, $n$ (\%)

Concomitant NSAIDs, $n$ (\%)

Both concomitant glucocorticoids and NSAIDs, $n$ (\%)

Concomitant aspirin, $n$ (\%)

Medical history of concurrent inflammatory bowel disease, $n$ (\%)

Diverticular problems (diverticulitis, diverticulum, diverticulosis), $n$ (\%)

No concurrent or medical history of diverticular disease, $n(\%)^{\mathrm{d}}$

Diverticulosis, $n$

Diverticulitis, $n$

Diverticular perforation, $n$

Diverticulosis and diverticular perforation, $n$

Diverticulitis and diverticular perforation, $n$

Diverticulosis, diverticulitis, and diverticular perforation, $n$

Concurrent diverticular disease, $n$ (\%)

Concurrent diverticulosis, $n$

Concurrent diverticulitis, $n$

Concurrent diverticulosis and diverticulitis, $n$

Medical history of diverticular disease, $n$ (\%)

History of diverticulosis, $n$

History of diverticulitis, $n$
Number/percentage/95\% CI

437

382,621

449

$1.2(1.1-1.3)$

36

32 (88.9)

$20(55.5)$

$18(50.0)$

$3(8.3)$

13 (36.1)

202

152 (75.2)

$96(47.5)$

78 (38.6)

17 (8.4)

$9(4.5)$

135 (66.8)

91 (45.0)

7

17

24

5

36

2

$22(10.9)$

14

4

4

23 (11.4)

14

7 
Table 3 continued

\begin{tabular}{ll}
\hline Patients treated with TCZ & Number/percentage/95\% CI \\
\hline History of both diverticulosis and diverticulitis, $n$ & 2 \\
History of GIP, $n(\%)$ & $3(1.5)$ \\
\hline
\end{tabular}

$C I$ confidence interval, GIP gastrointestinal perforation, NSAID nonsteroidal anti-inflammatory drug, $P Y$ patient-year, $T C Z$ tocilizumab

a As defined in each row

b Sufficient information for a comprehensive analysis was available for 298 GIP events reported in 295 patients. For 60 of these patients, the site of GIP was unspecified. Some patients had more than 1 site of perforation: 2 patients had both a lower GIP and an unspecified site of perforation; in 1 patient, only 1 GIP was reported, although in the case details both upper and lower GIPs were identified-these have been included as both upper and lower GIPs, respectively

${ }^{c}$ In one case, both medical history and concomitant condition of diverticular disease were reported

d In these 91 patients, the qualifying event was observed at the time of GIP diagnosis and was either diverticular perforation or the GIP was associated with diverticular disease (diverticulitis, diverticulum, diverticula)

perforation accompanied by both diverticulosis and diverticulitis).

Of 437 patients with GIP retrieved from the postmarketing safety database, 21 (4.8\%) had fatal outcomes. In 18 of these 21 patients, GIP was reported as the underlying event of death. Of these 18 patients, perforation occurred in the lower GI tract in 14 patients, the upper GI tract in 2 patients, and unspecified locations in 2 patients. In the remaining 3 patients, the causes of death were peritonitis secondary to perforation of colonic diverticulitis, perforative peritonitis and disseminated intravascular coagulation, and unrelated causes.

\section{US Insurance Claims Database}

A total of 58,334 patients with RA treated with an aTNF, abatacept, or TCZ who met the eligibility criteria for analysis were identified in the healthcare claims. Patients were followed for a mean of 535 days. Overall, approximately $50 \%$ of patients had no evidence of prior exposure to a qualifying biologic DMARD. However, of the patients who initiated TCZ, most (88\%) had prior biologic exposure; therefore, the safety analysis reported in detail here focused only on the population of patients who had received $\geq 1$ prior biologic DMARD.

Of the patients with prior biologic exposure, the mean age was similar for those who received an aTNF, abatacept, or TCZ (52.2, 54.4, and 54.1 years, respectively; Table 4). Patients who received TCZ tended to have received more prior biologics; $27.3 \%$ of patients in the TCZ group received $\geq 3$ prior biologics compared with $11.4 \%$ in the abatacept group and $7.8 \%$ in the aTNF group. Similar proportions of patients across the groups had exposure to prescription NSAIDs $(43.6 \%, 41.0 \%$, and $41.9 \%$ for the aTNF, abatacept, and TCZ groups, respectively). Mean exposure to oral glucocorticoids was also similar across groups.

Of the patients with prior biologic exposure, there were 21 GIPs (10 from the aTNF group, 5 from the abatacept group, and 6 from the TCZ group) per the specific definition and 31 (14 from the aTNF group, 8 from the abatacept group, and 9 from the TCZ group) per the sensitive definition (Table 5). The GIP incidence rate $(95 \% \mathrm{CI})$ per the specific definition for the TCZ group was $1.8(0.7-4.0)$ per 1000 PYs compared with $0.6(0.3-1.2)$ and $0.8(0.3-2.0)$ 
Table 4 Baseline demographic and clinical characteristics of patients with rheumatoid arthritis and prior biologic exposure from a healthcare insurance claims database

\begin{tabular}{|c|c|c|c|c|c|c|}
\hline \multirow[t]{2}{*}{ Characteristics } & \multirow{2}{*}{$\begin{array}{l}\text { Any aTNF } \\
(N=17,333)\end{array}$} & \multicolumn{3}{|c|}{ Selected aTNF ${ }^{a}$} & \multicolumn{2}{|c|}{ Other MOA agents } \\
\hline & & $\begin{array}{l}\mathrm{ADA} \\
(N=5765)\end{array}$ & $\begin{array}{l}\text { ETA } \\
(N=3675)\end{array}$ & $\begin{array}{l}\text { IFX } \\
(N=2339)\end{array}$ & $\begin{array}{l}\mathrm{ABA} \\
(N=6320)\end{array}$ & $\begin{array}{l}\text { TCZ } \\
(N=3602)\end{array}$ \\
\hline Female, $n(\%)$ & $13,762(79.4)$ & $4505(78.1)$ & $2932(79.8)$ & $1835(78.5)$ & $5190(82.1)$ & $2999(83.3)$ \\
\hline Age, mean (median), $n(\%)$ & $52.2(53)$ & $52.3(54)$ & $51.7(53)$ & $52.1(53)$ & $54.4(55)$ & $54.1(55)$ \\
\hline $65-74$ years & $1578(9.1)$ & $525(9.1)$ & $286(7.8)$ & $258(11)$ & $755(11.9)$ & $398(11)$ \\
\hline$\geq 75$ years & $660(3.8)$ & $231(4.0)$ & $126(3.4)$ & $95(4.1)$ & $362(5.7)$ & $183(5.1)$ \\
\hline \multicolumn{7}{|l|}{ Prior biologics, $n(\%)$} \\
\hline 1 & $12,687(73.2)$ & $5033(87.3)$ & $3098(84.3)$ & $1520(65.0)$ & $3392(53.7)$ & $1462(40.6)$ \\
\hline 2 & $3284(18.9)$ & $576(10.0)$ & $444(12.1)$ & $605(25.9)$ & $2210(35.0)$ & $1158(32.1)$ \\
\hline 3 & $943(5.4)$ & $117(2.0)$ & $92(2.5)$ & $155(6.6)$ & $573(9.1)$ & $642(17.8)$ \\
\hline 4 & $299(1.7)$ & $30(0.5)$ & $31(0.8)$ & $46(2.0)$ & $130(2.1)$ & $244(6.8)$ \\
\hline$\geq 5$ & $120(0.7)$ & $9(0.2)$ & $10(0.3)$ & $13(0.6)$ & $15(0.2)$ & $96(2.7)$ \\
\hline $\begin{array}{l}\text { Duration of prior biologic } \\
\text { exposure, mean (median), days }\end{array}$ & $540(316)$ & $545.3(304)$ & $422(238)$ & $464.9(282)$ & $643.3(402)$ & $713.2(483)$ \\
\hline csDMARDs, $n(\%)$ & $6021(34.7)$ & $1936(33.6)$ & $1266(34.4)$ & $867(37.1)$ & $2359(37.3)$ & $1324(36.8)$ \\
\hline NSAIDs, $n(\%)$ & $7550(43.6)$ & $2464(42.7)$ & $1603(43.6)$ & $1040(44.5)$ & $2592(41.0)$ & $1512(41.9)$ \\
\hline \multicolumn{7}{|l|}{ Oral glucocorticoids } \\
\hline$<7.5 \mathrm{mg} /$ day & $8270(47.7)$ & $2692(46.7)$ & $1789(48.7)$ & $1110(47.5)$ & $3228(51.1)$ & $1765(49)$ \\
\hline$\geq 7.5 \mathrm{mg} /$ day & $2156(12.4)$ & $683(11.8)$ & $465(12.7)$ & $367(15.7)$ & $926(14.7)$ & $647(18)$ \\
\hline Dose, mean (median), mg/day & $5.9(3.3)$ & $5.5(3.2)$ & $6.6(3.3)$ & $7.6(3.9)$ & $5.9(3.6)$ & $6.8(4.2)$ \\
\hline CCI, mean (median) & $2.3(2)$ & $2.2(2)$ & $2.3(2)$ & $2.3(2)$ & $2.5(2)$ & $2.6(2)$ \\
\hline
\end{tabular}

$A B A$ abatacept, $A D A$ adalimumab, aTNF anti-tumor necrosis factor, $C C I$ Charlson Comorbidities Index, $c s D M A R D$ conventional synthetic disease-modifying antirheumatic drug, ETA etanercept, IFX infliximab, $M O A$ mechanism of action, NSAID nonsteroidal anti-inflammatory drug, $T C Z$ tocilizumab

${ }^{a}$ These individual aTNF agents were selected to present because they represent the largest cohorts. The multivariable model used all five aTNF agents pooled vs. TCZ

per 1000 PYs for the aTNF and abatacept groups, respectively. According to the specific definition, the incidence rates (95\% CI) for the individual aTNF agents ranged from 0.4 (0.1-1.4) to $1.3(0.3-3.8)$ per 1000 PYs with widely overlapping CIs. According to the sensitive definition, the incidence rate $(95 \%$ CI) per 1000 PYs for the TCZ group was 2.8 (1.3-5.2) compared with $0.9(0.5-1.5)$ and 1.4
(0.6-2.7) for the aTNF (with widely overlapping CIs) and abatacept groups, respectively. The majority of GIPs $(71.4 \%)$ were reported in the lower GI tract, regardless of treatment or definition. The lower GI tract perforations were mostly associated with diverticular diagnoses, including three cases in the aTNF group, four in the abatacept group, and two in the TCZ group. 
Table 5 Incidence rates ${ }^{a}$ of GIPs using sensitive and specific definitions in patients with rheumatoid arthritis and prior biologic exposure from a healthcare insurance claims database

\begin{tabular}{|c|c|c|c|c|c|c|}
\hline \multirow[t]{2}{*}{ Treatment } & \multicolumn{3}{|c|}{ Specific definition ${ }^{b}$} & \multicolumn{3}{|c|}{ Sensitive definition ${ }^{c}$} \\
\hline & $\bar{n}$ & PYs & IR $(95 \% \mathrm{CI})$ & $\bar{n}$ & PYs & IR $(95 \% \mathrm{CI})$ \\
\hline \multicolumn{7}{|l|}{ Any aTNF } \\
\hline All GIP & 10 & 15,925 & $0.6(0.3-1.2)$ & 14 & 15,921 & $0.9(0.5-1.5)$ \\
\hline Lower GIP & 6 & 15,926 & $0.4(0.1-0.8)$ & 10 & 15,922 & $0.6(0.3-1.2)$ \\
\hline \multicolumn{7}{|l|}{ Etanercept } \\
\hline All GIP & 2 & 3237 & $0.6(0.1-2.2)$ & 3 & 3235 & $0.9(0.2-2.7)$ \\
\hline Lower GIP & 0 & 3238 & $0(0.0-1.1)$ & 1 & 3235 & $0.3(0.0-1.7)$ \\
\hline \multicolumn{7}{|l|}{ Adalimumab } \\
\hline All GIP & 2 & 5201 & $0.4(0.1-1.4)$ & 2 & 5201 & $0.4(0.1-1.4)$ \\
\hline Lower GIP & 1 & 5202 & $0.2(0.0-1.1)$ & 1 & 5202 & $0.2(0.0-1.1)$ \\
\hline \multicolumn{7}{|l|}{ Infliximab } \\
\hline All GIP & 3 & 2341 & $1.3(0.3-3.8)$ & 3 & 2341 & $1.3(0.3-3.8)$ \\
\hline Lower GIP & 3 & 2341 & $1.3(0.3-3.8)$ & 3 & 2341 & $1.3(0.3-3.8)$ \\
\hline \multicolumn{7}{|l|}{ Abatacept } \\
\hline All GIP & 5 & 5940 & $0.8(0.3-2.0)$ & 8 & 5938 & $1.4(0.6-2.7)$ \\
\hline Lower GIP & 5 & 5940 & $0.8(0.3-2.0)$ & 8 & 5938 & $1.4(0.6-2.7)$ \\
\hline \multicolumn{7}{|l|}{ Tocilizumab } \\
\hline All GIP & 6 & 3268 & $1.8(0.7-4.0)$ & 9 & 3267 & $2.8(1.3-5.2)$ \\
\hline Lower GIP & 5 & 3268 & $1.5(0.5-3.6)$ & 8 & 3267 & $2.5(1.1-4.8)$ \\
\hline
\end{tabular}

aTNF anti-tumor necrosis factor, $C I$ confidence interval, GIP gastrointestinal perforation, ICD-9-CM International Classification of Diseases, Ninth Revision, Clinical Modification, $I R$ incidence rate, $M O A$ mechanism of action, $P Y$ patient-year

${ }^{\text {a }}$ Incidence rates per 1000 PYs

b The specific definition included only inpatient admissions with evidence of perforation based on the presence of the word perforation in ICD-9-CM diagnosis descriptions for esophageal rupture; gastric, duodenal, peptic, or gastrojejunal ulcers; and unspecified GIP. Cases of appendicitis, diverticulitis, diverticulosis, or ischemic colitis associated with surgical GI procedures were not included

${ }^{c}$ The sensitive definition included any inpatient admissions with evidence of perforation based on (1) the presence of the word perforation in the following ICD-9-CM diagnosis descriptions: esophageal rupture; gastric, duodenal, peptic, or gastrojejunal ulcers; appendicitis; and GI perforation of an unspecific location in the large intestine or (2) an ICD-9-CM diagnosis of diverticulitis, diverticulosis, or ischemic colitis plus a Current Procedural Terminology code for suture or resection of the small or large intestine

The unadjusted absolute rate differences (95\% CIs) between the TCZ and aTNF groups for all GIPs were 1.2 (-0.3 to 2.5$)$ and $1.9(0.0-3.7)$ per 1000 PYs per the specific and sensitive definitions, respectively. For lower GIPs, the unadjusted absolute rate differences were $1.2(-0.2$ to 2.5$)$ and $1.8(0.1-3.6)$ per 1000 PYs per the specific and sensitive definitions, respectively. 
Multivariate analysis compared adjusted GIP incidence rates between aTNF and TCZ cohorts with prior exposure to $\geq 1$ biologic. The adjusted IRRs (95\% CI) for all GIPs in patients who received TCZ versus those who received an aTNF were 2.2 (0.7-6.6; specific definition) and 2.2 (0.9-5.4; sensitive definition). For lower GIP events, the adjusted IRRs in patients who received TCZ versus those who received an aTNF were 4.0 (1.1-14.1; specific definition) and 3.1 (1.1-8.4; sensitive definition). Both the number of prior biologics and the comorbidities index were significantly associated with the risk of GIP events [IRR: 1.5 (95\% CI, 1.0-2.2), $P=0.036$ and 1.2 (95\% CI, 1.0-1.4), $P=0.018$, respectively].

In the biologic-naïve populations, no patients treated with TCZ (12\% of the overall TCZ exposure) experienced a GIP; in patients treated with aTNFs (75-times greater patient exposure than for TCZ), the GIP incidence rate was similar to the overall aTNF-exposed population.

\section{DISCUSSION}

The incidence rates of GIP events in patients treated with TCZ are reported here from three distinct patient populations: patients from postmarketing, clinical trial, and real-world settings. GIP event rates in patients treated with TCZ ranged from 1.2 to 3.9 per 1000 PYs for the different populations and per definitions of a GIP event. In the TCZ-IV all-exposure RA clinical trials $(17,905.9$ PYs of TCZ exposure), the incidence rate of adjudicated GIP events was 1.9 per 1000 PYs; this rate did not increase over time and was consistent with the incidence rate in the TCZ-SC all-exposure RA clinical trial population. In the clinical trial population, the proportion of patients with a GIP event was similar between patients with prior DMARD use and those who were DMARD-naïve; similarly, no difference was observed between patients with prior aTNF use and those where were aTNF-naïve (data not shown). In the global postmarketing TCZ safety database $(382,621 \mathrm{PYs}$ of TCZ exposure), the reporting rate of GIP events was 1.2 per 1000 PYs. In the healthcare claims database TCZ population (3268 PYs of TCZ exposure), the incidence rate of GIP events ranged from 1.8 to 2.8 per 1000 PYs; the GIP incidence rate in the combined aTNF population (15,921-15,925 PYs of exposure) ranged from 0.6 to 0.9 per 1000 PYs. In all three TCZ populations examined here (clinical trial, postmarketing safety, and healthcare claims databases), the majority of GIPs occurred in the lower GI tract.

Our results are consistent with those reported in a recent analysis by Xie et al. [22] using data from MarketScan and Medicare healthcare claims from 2006 to 2014 of 190,061 patients with RA. Using the same sensitive definition as in our study, they reported an overall incidence rate of hospitalized GIP per 1000 PYs of 1.55 for TCZ, 1.29 for tofacitinib, 1.10 for abatacept, 0.73 for rituximab, and 0.84 for aTNFs (with similar incidence rates for adalimumab, etanercept, and infliximab).

Comparison of GIP incidence rates across biologic therapies for RA is limited by the ability to make comparisons across different data sets with different strengths, limitations, and patient populations. The healthcare claims database examined in this study provides real-world data within the same database on large cohorts of patients with RA who received biologic therapy. Of patients with prior biologic exposure, the demographics and baseline characteristics were mostly similar between those who received TCZ, aTNF, or abatacept therapy, except that patients treated with TCZ 
had received more prior biologic therapies compared with patients treated with aTNF agents. The rates of GIP were consistently higher in the TCZ cohort than in the aTNF cohort per both the specific and sensitive definitions. The absolute rate difference between TCZ and aTNFs for all GIPs was 1.2 per 1000 PYs per the specific definition and 1.9 per 1000 PYs per the sensitive definition, corresponding to numbers needed to harm (NNH) of 828 and 533, respectively. These NNHs suggest that, for every 533-828 patients treated with TCZ for 1 year, 1 additional GIP event might occur compared with patients treated with aTNF agents. Of the 471 biologic-naïve patients treated with TCZ, none experienced GIP, consistent with the observation that the number of prior biologics was associated with the risk of GIP. A robust unbiased comparison of the GIP incidence rates between TCZ and aTNFs in the biologic-naïve population is not possible due to the low exposure of biologic-naïve patients treated with TCZ compared with aTNFs.

Recently, data from the German biologics registry RABBIT (RA oBservation of Biologic Therapy) compared the incidence rate of lower GIPs in patients with RA treated with TCZ with those treated with other biologics [23]. The lower GIP event rate for patients treated with TCZ was 3.0 events per 1000 PYs compared with 0.5 events per 1000 PYs in patients treated with aTNF agents (with similar incidence rates between adalimumab, etanercept and infliximab). Considering the small number of patients treated with TCZ who experienced a lower GIP event (11 patients treated in the RABBIT registry and 5-8 patients from the healthcare claims analysis in this study), the rates of lower GIP events between the RABBIT analysis and the US healthcare claims database analysis appear comparable. Importantly, conclusions regarding the RABBIT analysis are limited in comparisons of incidence rates between each cohort without adjustment for differences in patient and disease characteristics and medication history.

The strengths of this analysis include the data source for the healthcare claims database, which is among the largest proprietary sample available in the US, making it well suited for the study of infrequently occurring outcomes such as GIPs. In addition, the study included patients from both commercial and Medicare databases, thus representing both younger and older patients in the US. The main limitation of this study was the small number of GIP events for analysis in both the healthcare claims and clinical trials database populations. Within the clinical trials database, the small number of events was due to both the low event rate and the limited overall exposure; given this limited exposure, the event rate was able to be estimated with relatively high precision. The addition of three recently completed randomized controlled trials (MEASURE [19], FUNCTION [13], and ADACTA [20]) increased the TCZ-IV all-exposure clinical trial population from 4009 as reported by Curtis et al. [14] in 2015 to 5185 patients reported here. A limitation in the healthcare claims database analysis here was that only information on prescription medication claims was obtained; therefore, use of NSAIDs and over-the-counter medications used to treat GI symptoms was not captured. Although it likely had an insignificant effect on the results, it is important to note that about $5 \%$ of patients in the postmarketing safety database had concurrent or a history of inflammatory bowel disease, whereas patients with inflammatory bowel disease in the healthcare claims database were excluded from the analysis. Due to variation in reporting sources and the nature of spontaneously 
reported events, baseline characteristics of the postmarketing database population could not be characterized. In addition, events from the postmarketing population may have been underreported due to the voluntary nature of spontaneous safety reporting.

\section{CONCLUSIONS}

The rates for the rare GIP event reported here from TCZ-IV clinical trials and the TCZ postmarketing database are consistent with those from previous reports and showed no increase over time; the vast majority of events occurred in the lower GI tract and in patients with underlying undiagnosed diverticulitis. The GIP event rates from clinical trials and postmarketing reports were consistent with rates from a real-world data source of US healthcare claims, suggesting that GIP events in real-world clinical practice reflect what has been observed in the clinical trial program. Absolute rate differences between the TCZ and aTNF cohorts from US healthcare claims suggest that, in patients with prior biologic exposure, for each additional 1000 patients treated with TCZ for 1 year, 1-2 additional GIP events might occur compared with patients treated with aTNFs. Due to the rareness of GIP and the limited TCZ exposure in biologic-naïve patients, joint analyses of large RA biologic registries may clarify the relative differences between individual biologics in biologic-naïve patients.

\section{ACKNOWLEDGMENTS}

Sponsorship and article processing charges for this study were funded by Roche. Support for third-party writing assistance for this manuscript, furnished by Ellen Mercado, PhD, of Health Interactions, was provided by $\mathrm{F}$.
Hoffmann-La Roche, Ltd. All named authors meet the International Committee of Medical Journal Editors (ICMJE) criteria for authorship for this manuscript, take responsibility for the integrity of the work as a whole, and have given final approval to the version to be published.

Disclosures. Sharareh Monemi, Erhan Berber, Khaled Sarsour, Kathy Lampl, and Kamal Bharucha are employees of Genentech, Inc. Jianmei Wang is an employee of Roche Products Ltd. Attila Pethoe-Schramm is an employee of F. Hoffmann-La Roche Ltd.

Compliance with Ethics Guidelines. This article is based on previously conducted studies, and does not involve any new studies of human or animal subjects performed by any of the authors.

Open Access. This article is distributed under the terms of the Creative Commons Attribution-NonCommercial 4.0 International License (http://creativecommons.org/licenses/ by-nc/4.0/), which permits any noncommercial use, distribution, and reproduction in any medium, provided you give appropriate credit to the original author(s) and the source, provide a link to the Creative Commons license, and indicate if changes were made.

\section{REFERENCES}

1. van der Kooij SM, de Vries-Bouwstra JK, Goekoop-Ruiterman YP, et al. Limited efficacy of conventional DMARDs after initial methotrexate failure in patients with recent onset rheumatoid arthritis treated according to the disease activity score. Ann Rheum Dis. 2007;66:1356-62.

2. Breedveld FC, Weisman MH, Kavanaugh AF, et al. The PREMIER study: a multicenter, randomized, double-blind clinical trial of combination therapy with adalimumab plus methotrexate versus methotrexate alone or adalimumab alone in 
patients with early, aggressive rheumatoid arthritis who had not had previous methotrexate treatment. Arthritis Rheum. 2006;54:26-37.

3. Emery P, Breedveld FC, Hall S, et al. Comparison of methotrexate monotherapy with a combination of methotrexate and etanercept in active, early, moderate to severe rheumatoid arthritis (COMET): a randomised, double-blind, parallel treatment trial. Lancet. 2008;372:375-82.

4. Smolen JS, Landewe R, Breedveld FC, et al. EULAR recommendations for the management of rheumatoid arthritis with synthetic and biological disease-modifying antirheumatic drugs: 2013 update. Ann Rheum Dis. 2014;73:492-509.

5. Smolen JS, Beaulieu A, Rubbert-Roth A, et al. Effect of interleukin-6 receptor inhibition with tocilizumab in patients with rheumatoid arthritis (OPTION study): a double-blind, placebo-controlled, randomised trial. Lancet. 2008;371:987-97.

6. Emery P, Keystone E, Tony HP, et al. IL-6 receptor inhibition with tocilizumab improves treatment outcomes in patients with rheumatoid arthritis refractory to anti-tumour necrosis factor biologicals: results from a 24-week multicentre randomised placebo-controlled trial. Ann Rheum Dis. 2008;67:1516-23.

7. Genovese MC, McKay JD, Nasonov EL, et al. Interleukin-6 receptor inhibition with tocilizumab reduces disease activity in rheumatoid arthritis with inadequate response to disease-modifying antirheumatic drugs: the tocilizumab in combination with traditional disease-modifying antirheumatic drug therapy study. Arthritis Rheum. 2008;58:2968-80.

8. Kremer JM, Blanco R, Brzosko M, et al. Tocilizumab inhibits structural joint damage in rheumatoid arthritis patients with inadequate responses to methotrexate: results from the double-blind treatment phase of a randomized placebo-controlled trial of tocilizumab safety and prevention of structural joint damage at one year. Arthritis Rheum. 2011;63:609-21.

9. Jones G, Sebba A, Gu J, et al. Comparison of tocilizumab monotherapy versus methotrexate monotherapy in patients with moderate to severe rheumatoid arthritis: the AMBITION study. Ann Rheum Dis. 2010;69:88-96.

10. Kivitz A, Olech E, Borofsky M, et al. Subcutaneous tocilizumab versus placebo in combination with disease-modifying antirheumatic drugs in patients with rheumatoid arthritis. Arthritis Care Res (Hoboken). 2014;66:1653-61.
11. Ogata A, Tanimura K, Sugimoto T, et al. Phase III study of the efficacy and safety of subcutaneous versus intravenous tocilizumab monotherapy in patients with rheumatoid arthritis. Arthritis Care Res (Hoboken). 2014;66:344-54.

12. Burmester GR, Rubbert-Roth A, Cantagrel A, et al. A randomised, double-blind, parallel-group study of the safety and efficacy of subcutaneous tocilizumab versus intravenous tocilizumab in combination with traditional disease-modifying antirheumatic drugs in patients with moderate to severe rheumatoid arthritis (SUMMACTA study). Ann Rheum Dis. 2014;73:69-74.

13. Burmester GR, Rigby WF, van Vollenhoven RF, et al. Tocilizumab in early progressive rheumatoid arthritis: FUNCTION, a randomised controlled trial. Ann Rheum Dis. 2016;75:1081-91.

14. Curtis JR, Perez-Gutthann S, Suissa S, et al. Tocilizumab in rheumatoid arthritis: a case study of safety evaluations of a large postmarketing data set from multiple data sources. Semin Arthritis Rheum. 2015;44:381-8.

15. Del Valle J. Peptic ulcer disease and related disorders. Harrison's principles of internal medicine, 18 edn. New York: McGraw-Hill; 2012. p. Ch. 293.

16. Myasoedova E, Matteson EL, Talley NJ, Crowson CS. Increased incidence and impact of upper and lower gastrointestinal events in patients with rheumatoid arthritis in Olmsted County, Minnesota: a longitudinal population-based study. J Rheumatol. 2012;39:1355-62.

17. Curtis JR, Lanas A, John A, Johnson DA, Schulman KL. Factors associated with gastrointestinal perforation in a cohort of patients with rheumatoid arthritis. Arthritis Care Res (Hoboken). 2012;64:1819-28.

18. Curtis JR, Xie F, Chen L, et al. The incidence of gastrointestinal perforations among rheumatoid arthritis patients. Arthritis Rheum. 2011;63:346-51.

19. McInnes IB, Thompson L, Giles JT, et al. Effect of interleukin-6 receptor blockade on surrogates of vascular risk in rheumatoid arthritis: MEASURE, a randomised, placebo-controlled study. Ann Rheum Dis. 2015;74:694-702.

20. Gabay C, Emery P, van Vollenhoven R, et al. Tocilizumab monotherapy versus adalimumab monotherapy for treatment of rheumatoid arthritis (ADACTA): a randomised, double-blind, controlled phase 4 trial. Lancet. 2013;381:1541-50.

21. Curtis JR, Chen SY, Werther W, John A, Johnson DA. Validation of ICD-9-CM codes to identify 
gastrointestinal perforation events in administrative claims data among hospitalized rheumatoid arthritis patients. Pharmacoepidemiol Drug Saf. 2011;20:1150-8.

22. Xie F, Yun H, Bernatsky S, Curtis J. Risk for gastrointestinal perforation among rheumatoid arthritis patients recieving tofacitinib, tocilizumab, or other biologics. Arthritis
Rheumatol. 2016. doi:10.1002/art39761 [Epub ahead of print].

23. Strangfeld A, Richter A, Herzer P, et al. Risk for lower perforations in RA patients treated with tocilizumab in comparison to treatment with TNF inhibitors, rituximab, abatacept or conventional synthetic DMARDs [oral presentation]. Arthritis Rheumatol. 2015;67(Suppl):S10. 